\title{
Wann Diabetiker trainieren sollten
}

Für Typ-2-Diabetiker ist es möglicherweise günstiger, das tägliche Bewegungspensum nicht auf einmal zu absolvieren, sondern auf die Zeit nach den Hauptmahlzeiten zu verteilen.

Leitlinien zur körperlichen Aktivität bei Typ-2-Diabetes sehen eine Mindestzeit von 150 Minuten pro Woche vor; zum Zeitpunkt des Trainings machen sie keine Angaben. Das könnte nach den Erkenntnissen neuseeländischer Ärzte ein Versäumnis sein. Sie haben in einer randomisierten Cross-over-Studie festgestellt, dass die postprandialen Blutzuckerspiegel niedriger bleiben, wenn die Patienten nach jeder der drei Hauptmahlzeiten jeweils 10 Minuten lang gehen anstelle einmal täglich 30 Minuten lang spazieren zu gehen [1].

41 Typ-2-Diabetiker wurden für die Untersuchung nach dem Zufallsprinzip der 1x30- oder der 3x10-Minuten-Bewegungsgruppe zugeteilt; in Letzterer sollten sie jeweils innerhalb von fünf Minu- ten nach beendetem Essen mit dem Gehen beginnen. Nach zwei Wochen folgte eine Auswaschphase von 30 Tagen, danach wurde das Regime getauscht. Eine kontinuierliche Messung des Blutzuckers erfolgte jeweils an den Tagen 8-14 der Intervention.

\section{Stärkster Effekt nach dem Abendesssen}

Die Zunahme der Fläche unter der Blutzuckerkurve (incremental area under the curve, iAUC) in den ersten drei Stunden nach einer Hauptmahlzeit, der primäre Studienendpunkt, fiel mit der postprandialen Bewegung um 12\% geringer aus. Verantwortlich dafür war v. a. die hochsignifikante Reduktion des iAUC nach dem Abendessen um 22\%. Dass der Effekt zur letzten Mahlzeit des Tages am stärksten war, könnte daran liegen, dass dabei die meisten Kohlenhydrate konsumiert wurden. Der mittlere Blutzuckerspiegel in den drei Stunden danach lag mit postprandialer Bewegung im Mittel um 0,5 mmol/l unter dem der Vergleichsgruppe. Die Kohlenhydratzufuhr insgesamt unterschied sich zwischen den Gruppen nicht.

Der postprandiale Blutzuckerspiegel ist ein unabhängiger kardiovaskulärer Risikofaktor. Die Studienautoren um Andrew N. Reynolds von der Universität in Dunedin sehen deswegen Evidenz für eine Ergänzung der Leitlinien. "Sie sollten spezifisch zu postprandialer Aktivität raten, besonders nach Mahlzeiten mit viel Kohlenhydraten." Durch das geeignete Timing der körperlichen Aktivität könne vermutlich (Mahlzeiten-)Insulin eingespart werden.

(Beateschumacher)

Reynolds A.N. et al. Diabetologia 2016;59(12):2572-78

\section{Gefahr aus dem sozialen Umfeld}

\section{Typ-2-Diabetes ist ansteckend}

Ob jemand an Diabetes erkrankt oder nicht, hängt auch von den Menschen ab, die ihm nahestehen.

US-Mediziner werteten im Rahmen einer retrospektiven Analyse Daten von 4797 Teilnehmern der Framingham Off-

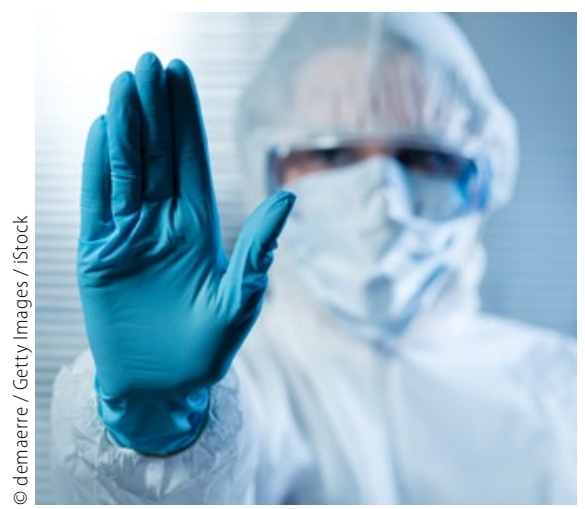

Schutzkleidung verhindert den Diabetes leider nicht. spring Study (FOS) aus, die zwischen 1971 und 2008 erhoben worden waren. Die Daten beinhalten Angaben zur kardiometabolischen Gesundheit der Studienteilnehmer sowie von engen Kontaktpersonen. Ein Typ-2-Diabetes (T2D) bestand bei 136 Teilnehmern schon zum Studienstart, bei 692 wurde er in der Studie diagnostiziert.

\section{Adipositas des Ehepartners erhöht das Diabetesrisiko}

Wenn enge Beziehungen zu Patienten mit Adipositas bzw. mit T2D angegeben wurden, erhöhte dies die Wahrscheinlichkeit einer späteren Diabetesdiagnose signifikant um 32\% bzw. um 21\%. Eine Aufschlüsselung nach der Art der Beziehung ergab, dass bei einer Adipositas des Ehe- partners das Diabetesrisiko um 54\% erhöht war. Starkes Übergewicht bei Geschwistern zeigte dagegen keinen Einfluss. Umgekehrt war ein T2D bei Schwester oder Bruder, aber nicht beim Ehepartner, mit einem höheren Erkrankungsrisiko verbunden; die Risikosteigerung lag bei $64 \%$. Das zusätzliche Risiko bestand auch noch, wenn genetische Veranlagungen berücksichtigt wurden.

„Wir gehen davon aus, dass die Assoziationen zustande kommen, weil die Beteiligten dasselbe Gesundheitsverhalten haben, v. a. in Bezug auf Ernährung und Sport", schreiben die Studienautoren. Informationen über nahestehende Personen könnten dazu beitragen, Patienten mit hohem T2D-Risiko früher zu erkennen und präventive Maßnahmen einzuleiten.

(Beate Schumacher)

Raghavan Set al. J Gen Intern Med 2016;31(10):1127-33 